\title{
El Castillo de Cullera. Adecuaciones del castillo islámico
} \author{
José Manuel Climent Simón a , Maria Isabel Giner García ${ }^{b}$, Ángeles Rodrigo Molina ${ }^{c}$ \\ ${ }^{a}$ Dep. Proyectos arquitectónicos ETSAV. UPV, Valencia, España, jmcliment@gmail.com, ${ }^{b}$ Dep. Construcciones \\ Arquitectónicas ETSIE, UPV. Valencia, España, magigar@csa.upv.es, ${ }^{\mathrm{c}}$ Dep. Expresión Gráfica Arquitectónica \\ ETSIE, UPV, Valencia, España, arodrigo@ega.upv.es
}

\begin{abstract}
The strategic location of most of the fortifications has led to the use throughout history of many settlements, carrying out numerous renovations to adapt them to the defense needs of each period.

The privileged location of the Cullera Castle, from which a vast territory south to the Júcar river including coastal and river areas can be controlled, has justified that from its origins in the tenth century to the mid-eighteenth century it would be used as a fortified enclosure.

Due to its long history, this defensive enclave boasts numerous examples of architectural elements from different ages. Therefore, it constitutes, a wealth of great historical interest, highlighting structures dating from Islamic Period, from which numerous remains have been preserved. Meanwhile, subsequent renovations following the Christian reconquest, call attention to the last structures built during the Carlist Wars. Since its desertion after the last armed conflicts, the Castle and its enclosures entered a stage of almost total abandonment, reaching modern times in an advanced state of ruin.

In the last decade there have been a series of interventions following a sequence of stages included in a Master Plan which has resulted in the consolidation and restoration of the different structures of the ensemble, including the necessary archaeological research to pursue each of them.

Buildings' reuse as a guarantor of its maintenance and conservation was one of the intervention approaches, so that different socio-cultural uses compatible with the nature of the monument were proposed, contributing at the same time to its dissemination.
\end{abstract}

Keywords: conservación, reutilización, difusión, estrategias

\section{Introducción}

En el marco de este Congreso, dedicado a las Fortificaciones de la Edad Moderna en la costa oeste del Mediterráneo, nuestra aportación pretende abarcar varios aspectos. En primer lugar realizar una descripción actualizada de las estructuras defensivas del Castillo de Cullera, incluyendo una breve reseña histórica, así como un estudio constructivo. En segundo lugar se pretende mostrar de forma resumida el alcance de las intervenciones realizadas entre los años 2005 y 2012, siguiendo las indicaciones y el plan de etapas recogidos en el documento "Plan Director de Actuaciones del Castillo de Cullera y su entorno", para finalmente, a modo de conclusión, realizar una valoración de las actuaciones realizadas, relacionando estas con la recuperación y puesta en valor del monumento y su uso y disfrute por la ciudadanía.

\section{Descripción del Castillo}

El Castillo de Cullera se ubica en la ladera meridional de la montaña de Cullera o Montaña del Oro.

La ubicación del mismo, como en la mayoría de casos, responde a criterios geoestratégicos, ya 
que desde este emplazamiento se controla un amplio territorio de costa y llano litoral, especialmente al sur del rio Júcar, teniendo comunicación visual con los castillos de Corbera y Bairén (Gandia)

Aunque los trabajos de arqueología realizados han datado materiales pertenecientes a la cultura Ibérica de los siglos V y VI a.C, el origen del castillo, tal y como ahora lo conocemos, se remonta al s.X, siendo las primeras construcciones de época califal. A partir de este momento, el conjunto fortificado ha sido objeto de numerosas transformaciones a lo largo de su dilatada historia de más de 1000 años.

Los principales episodios en la historia del conjunto se corresponden con el periodo Almohade, s.XII y XIII.

Desde la conquista cristiana del mismo en 1.238 no re reconocen importantes transformaciones del mismo hasta los siglos XV-XVI, periodo en el que se realizan obras que cambiarán el aspecto de la fortaleza islámica, como son la sala gótica, sala de armas y posteriormente el baluarte defensivo adosado a la Torre Major.

El último periodo de transformaciones importantes tiene lugar en el s. XIX, coincidiendo con las Guerras Carlistas, equipando parte de sus defensas con aspilleras adaptadas a la fusilería.

Desde este momento el Castillo deja de utilizarse y por tanto de mantenerse hasta que se instala una compañía de padres Franciscanos, siendo su último uso hacia los años $60 \mathrm{del}$ pasado siglo como escolanía.

A pesar de las numerosas transformaciones realizadas en el mismo, el conjunto conserva gran parte de su estructura inicial, correspondiente con la construcción islámica entre los siglos X-XIII.

Dicha estructura sigue el esquema tripartito utilizado en otros recintos próximos como puede ser el Castillo de Bairén en Gandía.

De manera que se pueden distinguir claramente tres recintos fortificados (Fig. 1):
En la zona más alta se sitúa la llamada Alcazaba o Fortaleza, junto a éste un primer recinto amurallado que denominamos Primer Albacar y ocupando gran parte de la ladera se extiende el gran recinto del Segón Albacar.

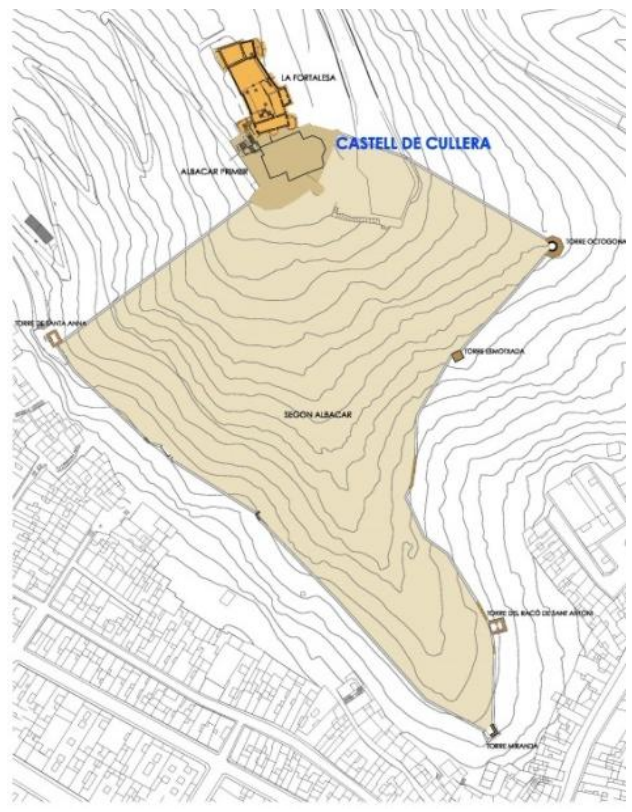

Fig. 1- Plano general de los recintos fortificados del Castell de Cullera. (Climent, J.M.)

El recinto superior presenta un patio en torno al cual se distribuyen los principales elementos entre los que destaca el gran volumen de la Torre Celoquia o Torre Major. El resto del recinto conserva parte de los elementos iniciales y numerosas reformas de épocas posteriores entre las que destaca la Sala Gótica.(Fig. 2)

Los Albacares eran recintos cerrados protegidos por lienzos y torres que suponían una primera línea de defensa y un espacio en el que albergar y refugiar a la población y sus pertenencias durante episodios de conflictos bélicos.

Esta zona del castillo es la peor conservada. En el caso del Primer Albacar por la construcción en su interior a finales del s. XIX del Santuario de la Virgen de la Encarnación y en el caso del Segón Albacar por los escasos restos que quedan en pie, ya que salvo las torres y algún tramo de lienzo el resto fue ordenado derribar en el $\mathrm{s}$. XVIII. 


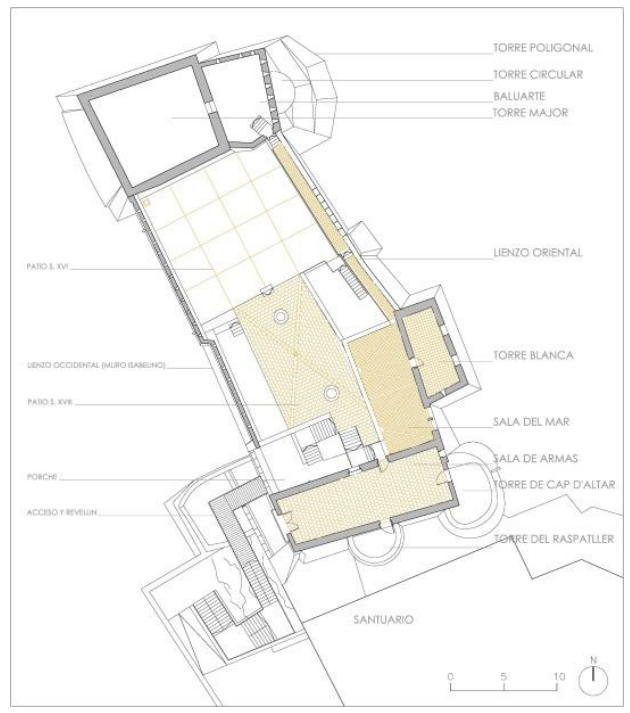

Fig. 2- Planta del nivel superior del recinto de la Fortaleza. (Climent, J.M.)

\subsection{Reseña histórica}

El Castillo de Cullera en la situación que ocupa en la actualidad, es una construcción de época califal (siglo X), erigida por el Estado cordobés para el control y defensa del territorio, especialmente la zona litoral y desembocadura del río Xúquer.

A comienzos del siglo XI formará parte del sistema defensivo, frontera meridional, de la taifa independiente de Valencia. En la primera mitad del siglo XII el territorio quedará incluido en la órbita política del imperio almorávide y después, en el imperio almohade, disfrutando de ciertos periodos de independencia.

El castillo pasó a manos cristianas poco después de la conquista de la ciudad de Valencia en 1238. Perteneció a la Orden del Hospital, y en la guerra de los dos Pedros del siglo XIV parece que una buena parte del mismo se derribó y volvió a construir y hasta el siglo XVI sufrió un largo periodo de decadencia y abandono.

En el siglo XV I se "moderniza" el castillo y se construyen una serie de baluartes y revellines, reforzando lienzos de muralla con alambores y disponiendo artillería en diferentes torres.
Todas estas obras, junto a la construcción de las torres vigía del litoral y el refuerzo de la iglesiafortaleza de la villa y el posterior construcción del recinto amurallado urbano, iban encaminadas a reforzar la defensa de la villa y el litoral frente a ataques y saqueos de la piratería.

En el siglo XIX volverá a registrarse cierta actividad militar con la guerra del francés y posteriormente con las guerras carlistas, construyéndose, principalmente, una serie de aspilleras, tanto en el lienzo occidental como en la Torre Major.

La presencia de un ermitaño que atendía la capilla de la Mare de Deu del Castell fue la causa de que el castillo se mantuviera más o menos en píe. Posteriormente, en la segunda mitad del siglo XIX se instaló una comunidad de frailes (Padres Franciscanos), construyéndose un santuario justo al lado del castillo.

\subsection{Estudio constructivo}

La característica principal de cualquier fortaleza es su función como reducto defensivo, por lo que todas ellas presentas un aspecto sólido y resistente; prueba de ello es la gran durabilidad de sus estructuras, que sin apenas mantenimiento han llegado a nuestros días con cierta dignidad.

Desde el punto de vista constructivo, en un conjunto de las dimensiones del castillo de Cullera, formado por varios recintos y teniendo en cuenta su dilatada historia, es normal encontrar un amplio elenco de soluciones y técnicas constructivas que abarcan desde las fábricas de mampostería armadas con emparrillados de madera, la construcción de torres de planta octogonal con la técnica del tapial calicostrado o la construcción de la sala gótica con bóvedas nervadas realizadas en sillería.

El conjunto de la Torre Major del Castell y el Baluarte defensivo situado en el acceso a la misma son un ejemplo en el que se puede recorrer la historia del Castillo, localizando en el mismo desde las primeras construcciones del siglo $\mathrm{X}$, hasta las últimas adaptaciones defensivas realizadas en el castillo, de mediados del siglo XIX que se corresponden con las 
defensas realizadas durante las Guerras Carlistas.

La Torre Major es uno de los elementos más representativos del castillo de Cullera. De planta cuadrada algo irregular y unas dimensiones en la base de unos 15 metros, se levanta hasta una altura máxima de 17 metros presentando un importante derrame en alguno de sus muros, ofreciendo un perfil ligeramente truncado.

De los antiguos niveles cubiertos con bóvedas no queda ningún resto, presentándose la torre totalmente vacía en su interior, donde es posible apreciar las diferentes etapas de su evolución mediante la lectura de las diferentes estructuras superpuestas que configuran la actual torre.

La construcción más antigua se trata de una torre construida con mampostería tomada con mortero de cal y armada con un entramado de rollizos de madera que se disponen en las caras interiores y exteriores en hiladas horizontales y que son unidas con otras piezas verticales más cortas. Estos dos entramados superficiales están unidos mediante otros elementos de madera que atraviesan el espesor del muro. Las diferentes piezas están unidas mediante grandes clavos metálicos, de los que se conserva algún ejemplar. Este tipo de estructura, datado en el siglo $X$, supone un interesante ejemplo de técnica constructiva por la utilización de la madera de esta forma tan característica en la construcción de muros de mampostería.

La utilización de secciones de madera de buena calidad, que han perdurado hasta nuestros días, en una zona geográfica donde no existen masas forestales para proveer dicha materia prima, se explica por la presencia del rio Xúquer y su desembocadura en el mar Mediterraneo. Por el río bajaban los troncos de conífera desde las zonas forestales del interior para ser embarcados y llevados a Denia y ser utilizada en la construcción de embarcaciones en las atarazanas de esta población.

La presencia de refuerzos con madera, aunque menos aparente que en este caso, se localiza también en otros elementos defensivos del castillo como las torres circulares del frente meridional de la fortaleza superior (Torre de Cap d'Altar y Torre del Raspatller)

Gran parte del muro meridional se desmoronó, permaneciendo sus restos en el interior de la torre hasta que se realizaron las primeras campañas de excavaciones arqueológicas.

Sobre los muros de la torre inicial, y adosándose exteriormente a ellos en tres de sus caras, se produce otra importante fase constructiva, datada en el s. XII, en la que se forra la antigua torre y se eleva por encima de la construcción. En este caso se emplea la técnica del tapial de mampuesto y argamasa de cal, muy visible en la cara norte.

En la siguiente intervención importante, datada en el s. XVI, se construyen los alambores y baluarte defensivo de la cara este ( Fig. 3 y 4), así como remates almenados que después serian eliminados por otras intervenciones posteriores. Es posible que en esta época se macizara de tierra en interior de la torre, adaptando su defensa a las nuevas técnicas bélicas. Los materiales empleados en esta fase son en general de peor calidad, estando constituidos por obra de mampostería trabada con morteros de cal, siendo en algunos casos como en los alambores del lienzo occidental de mampuesto y tierra sin apenas ligantes y recubiertos con una capa de mortero.

La última intervención de importancia tuvo lugar en el s. XIX con motivo de las Guerras Carlistas. Se recreció la torre con el actual cuerpo de aspilleras que remata el elemento en sus cuatro fachadas, se construyeron dos zonas cubiertas sobre la terraza y posteriormente en la cara occidental la espadaña que actualmente remata dicha fachada.

El Baluarte: La actual construcción está datada en el s. XVI y está formado por un cuerpo macizo situado junto al cara oriental de la Torre Major, desde donde se accede a ésta y al que se llega a través del "carreró" o paso de ronda del lienzo oriental tras salvar mediante una escalera el desnivel existente entre ambos. Tiene unas dimensiones aproximadas en la base de 12 x 10 metros y una altura total de unos 15 metros, 
presentando en su terraza superior unas dimensiones de 7 x 5 metros

$\mathrm{Su}$ construcción tenía como finalidad proteger del fuego de artillería a la torre por ese flanco oriental, así como utilizarlo para disponer la artillería defensiva.

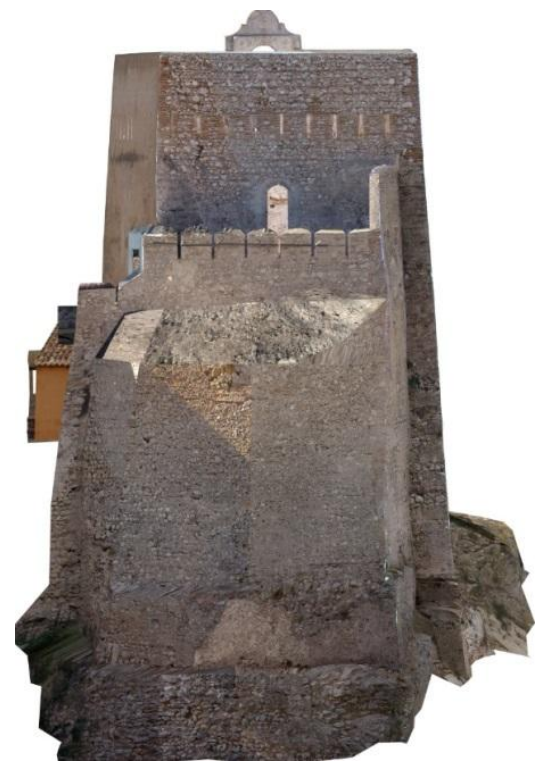

Fig. 3- Ortofotografía del Baluarte de defensa del acceso a la Torre Major (Global Mediterranea)

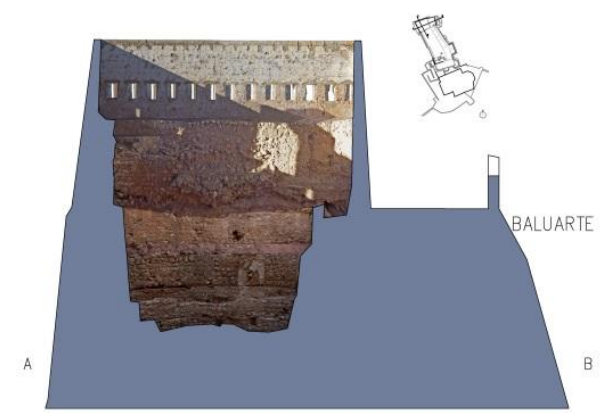

Fig. 4- Ortofotografía de la sección transversal de la Torre Major y Baluarte (Global Mediterranea)

Siguiendo los cánones de este tipo de estructuras defensivas, se trata de una estructura totalmente maciza con sus caras facetadas en forma poligonal para absorber y repeler los impactos de la artillería. A nivel de la terraza presenta una planta trapezoidal y está coronada con aspilleras y almenas en su cara oriental, con un muro más alto provisto de tres aspilleras en su cara norte y por otro muro donde se sitúa el acceso desde el carreró y varias aspilleras en su muro recayente al sur, estando protegido por la propia Torre Major por su lado oeste.

\section{Trabajos de recuperación y puesta en valor}

\subsection{El Plan Director de Actuaciones}

Tras varios años cerrado al público por el avanzado estado de ruina que presentaba, el Ayuntamiento de Cullera, consciente de la necesidad de recuperar la principal pieza de su patrimonio, promovió la implicación de la Generalitat Valenciana para iniciar las actuaciones en el Castillo.

Como resultado, en 2005 se recibió por parte de la Consellería de Educación, Cultura y Deporte el encargo para redactar el documento "Plan Director del Castell de Cullera y su entorno"

Debido a las características particulares de este tipo de edificaciones históricas de gran complejidad histórica y constructiva, en cuya restauración intervienen diferentes disciplinas y oficios, se hace necesario disponer de un instrumento que sea capaz de recoger toda esta complejidad y de forma coordinada establezca estrategias tendentes a la recuperación del conjunto.

En este sentido, el Plan Director se convierte en el Marco de Referencia que hace partícipes a todos los futuros agentes intervinientes y en el que se fijan las estrategias y planificaciones tendentes a la investigación, consolidación, restauración y puesta en valor del monumento.

Como en la gran mayoría de documentos de estas características, el objetivo a alcanzar es doble, por un lado se trata de planificar las actuaciones tendentes a la recuperación del conjunto monumental y su puesta en valor, y por otra de planificar los futuros usos y su gestión.

En la redacción del documento han participado diferentes profesionales tales como arquitectos, arquitectos técnicos, arqueólogos, historiadores, restauradores, etc. Así como técnicos responsables a nivel local y autonómico tales como las concejalías de Cultura y Turismo del 
Ayuntamiento de Cullera y la Consellería de Cultura.

Tras el análisis y síntesis realizados, se propone una secuencia espacio temporal de actuaciones, con un horizonte próximo como objetivo para cumplir las previsiones del plan.

Para ello se elabora un Plan de etapas basado en posibilidades reales en cuanto a su fragmentación presupuestaria, atendiendo también otras exigencias planteadas.

\subsection{Actuaciones de consolidación y restauración}

Siguiendo la planificación establecida en el Plan Director se fueron realizando la práctica totalidad de las actuaciones previstas en el mismo.

Las primeras fases se centraron en el recinto superior para pasar a intervenir posteriormente los restos del Segón Albacar.

En todas las actuaciones realizadas se ha trabajado conjuntamente con arqueólogos, que previamente y en paralelo al desarrollo de los trabajos realizaron importantes estudios $\mathrm{y}$ nuevas aportaciones que han servido para disponer de una actualizada y más completa documentación sobre los diferentes aspectos relacionados con la historia y construcción del castillo de Cullera.

Otro de los objetivos marcados a priori era la reutilización de parte de los espacios recuperados, de manera que en las diferentes fases se ha tenido en cuenta esta premisa, permitiendo compatibilizar la naturaleza monumental del conjunto con determinados usos de tipo socio-cultural, posibilitando en la actualidad que se desarrolle en el castillo un importante programa cultural que entre otras actividades incluye la exposición permanente del Museu Arqueológic de Cullera.

\section{Conclusiones}

La consolidación y restauración de las estructuras del castillo de Cullera no tendrían sentido sin el valor añadido de la información obtenida durante el transcurso de las actuaciones realizadas.

Durante todo el proceso se ha contado con la participación de diferentes disciplinas tales como historiadores, arqueólogos, restauradores, etc..., que a través de sus estudios han aportado importes novedades que completan y amplían el grado de conocimiento sobre el origen $y$ evolución de un conjunto edificado de una gran complejidad.

En este sentido cabe citar entre otras la aparición del segundo aljibe en el patio de armas, perfectamente conservado, los niveles originales del patio correspondientes al s. XVI, la existencia de dos troneras en dicho muro occidental, la existencia de una torre poligonal de tapia de época almohade que forraba otra anterior circular de mampostería y que a su vez son desmochadas y rodeadas por el baluarte del siglo XVI, la aparición de la cubierta original sobre canes de la sala de Armas, etc. Estos son sólo unos pocos ejemplos, quizá los más significativos, de entre otros muchos que han contribuido a enriquecer el conocimiento sobre este interesante ejemplo de arquitectura defensiva.

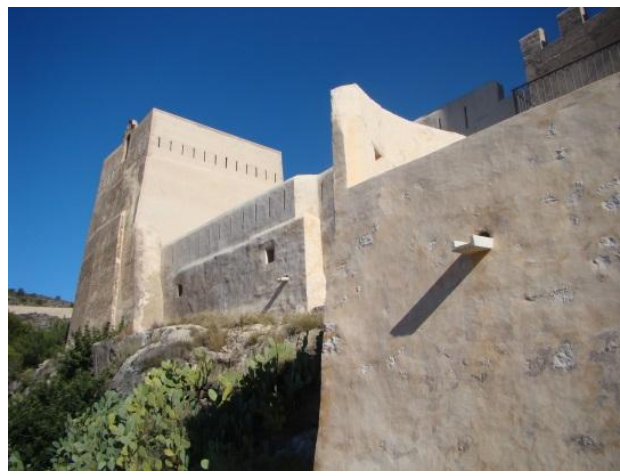

Fig. 5- Fachada occidental de la Fortaleza, en primer término los restos del Revellín del siglo XVI que defendía el acceso. Al fondo la Torre Major con el remate aspillerado del siglo XIX.

Centrando de nuevo la atención en la torre Major y Baluarte cabría reseñar que durante las intervenciones de restauración llevados a cabo en esta zona, los estudios arqueológicos realizados confirmaron la presencia de una 
construcción anterior que estaba en parte oculta por la obra del baluarte. Se trata de una torre poligonal construida con la técnica del tapial calicostrado que envuelve a una construcción anterior, formada por una torre de planta circular levantada con mampostería. Tanto esta primera torre de época islámica, como el forro poligonal de tapial de época almohade, como la construcción del Baluarte del s. XVI tenían como objeto la defensa del principal elemento del castillo que no era otro que su torre Major, más concretamente el frente oriental que alberga el acceso a la misma.

La construcción poligonal del s. XII-XIII es un caso similar al de la llamada Torre Octogonal, situada en una cota inferior, en el recinto del Segundo Albacar.

Todo ello supuso, además de una gran satisfacción por el hallazgo, un reto por la dificultad añadida a la hora de plantear la restauración del Baluarte, proponiendo que la presencia de todas estas fases constructivas contribuyera a la comprensión el proceso evolutivo de este sector del castillo, consecuencia de sucesivas superposiciones.

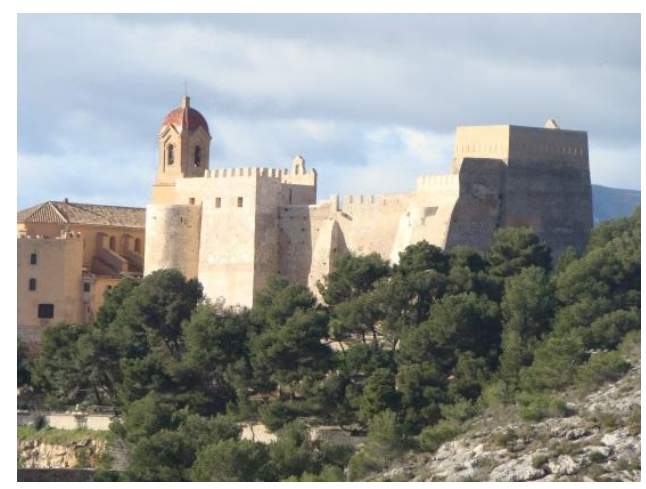

Fig. 6- Fachada oriental de la Fortaleza, a la derecha e Baluarte defendiendo el acceso a la Torre Major

\section{Referencias}

Arciniega García, L. (1997). El Castillo de Cullera. Informe histórico analítico. Universitat de Valencia.

Arciniega García, L. (2003). Sistemas de defensa en Cullera: Castillo, murallas y torres. Ajuntament de Cullera.

Climent Simón, J. M. (2005). Plan Director de actuaciones en el Castell de Cullera y su entorno, Conselleria de Educació, Cultura y Esport.

Cotino, F. (2002) "El castell de Cullera: interpretación de ciertas estructuras arquitectónicas fechables poco antes de la época de la conquista" en Castells, torres i fortificacions en la Ribera del Xúquer (A.Furió, J. Aparici, eds.). VIII Asemblea d'Història de la Ribera (Cullera 2000). Ajuntament de Cullera-Universitat de València, 129-132.

Díaz Borrás, A. (1988). "El asalto berberisco a Cullera en 1503 y Alzira en la defensa de la Ribera" en Al-Gezira 4/5, Alzira, 147-171.

López Elum, P. (1991). "La intervención y conquista de los castillos de Cullera y Bairén en el año 1239" en Cullaira 3, Cullera, 71-80.

Monraval Sapiña, M. (1990). "Torre Major del Castell. Cullera, la Ribera" en Excavacions arqueològiques de salvament a la Comunitat Valenciana 1984-1988. Vol. II. Intervencions Rurals, pp. 144-147.

Piles, A. (1893). "Historia de Cullera". Ajuntament de Cullera. 1979.

Vercher, S. (2005). "El Castell de Cullera a través dels memorials de l'any 1537" en Qulayra, Revista d'arqueologia i estudis històrics. I. Museo d'història i arqueología de Cullera. 
